\title{
3D Building Change Detection from High Resolution Spaceborne Stereo Imagery
}

\author{
Jiaojiao Tian, Houda Chaabouni-Chouayakh, Peter Reinartz \\ German Aerospace Center (DLR), Remote Sensing Technology Institute, 82234 Wessling, Germany \\ (Jiaojiao.Tian, Houda.Chaabouni, Peter.Reinartz)@dlr.de
}

\begin{abstract}
In this paper, a novel approach for 3D building change detection is proposed using Digital Surface Model (DSM) generated from High spatial Resolution Spaceborne Stereo (HRSS) imagery. To improve the change detection performance, the difference image is denoised by the detected shadow mask and DSM hole mask. Several thresholding algorithms are compared to remove spurious change in altitude caused mainly by computation errors in the DSM generation procedure. After applying the thresholding methods, object-oriented image analysis approach is carried out by comparing the size and shape properties of the objects that are extracted from the thresholded difference image to filter small changed regions. The performance evaluation of the proposed 3D building change detection confirms the effectiveness of this approach.
\end{abstract}

Keywords-component; 3D; change detection; DSM; HRSS imagery, building

\section{INTRODUCTION}

Detecting and monitoring urban area changes are of great relevance for city planning, disaster mitigation, environmental monitoring and military intelligence [1]. The most important objects in the field of urban area change detection are buildings. However, many changes of buildings can not be correctly detected in urban areas due to the fact that they share similar texture characteristics with other constructions. Exemplarily, buildings with gray roofs could be confused with streets when spectral-based building detection is adopted. The increasing availability of High Spatial Resolution Spaceborne Stereo (HRSS) imagery as well as the steady development of automatic DSM generation techniques [2-5], has created new alternatives for monitoring urban area changes. Indeed, by using the height change information extracted from DSMs, 3D changes can be detected.

In this paper, we focus on the automatic detection of new building constructions in urban areas. By using the difference based change detection methodology [6-8], our work is divided into 4 parts. The first part is the DSM coregistration. The second step is the denoising of the difference image with the detected shadow mask and DSM holes mask. In the following, we eliminate the undesired low height change areas in the difference image using several thresholding techniques. Finally, the change detection procedure is accomplished by comparing the size and shape properties of changed objects extracted from the thresholded difference image. At the end of this paper, we evaluate the performance of the change detection methods at pixel as well as object levels.

\section{DATASET}

This paper focuses on urban area 3D change detection by using the HRSS imagery of two dates (approximately 4 years difference) and the DSMs generated from them. The available ground truth concerning the changed buildings, extracted by visual/manual interpretation, is used to assess the change detection results.

\section{A. HRSS Imagery Description}

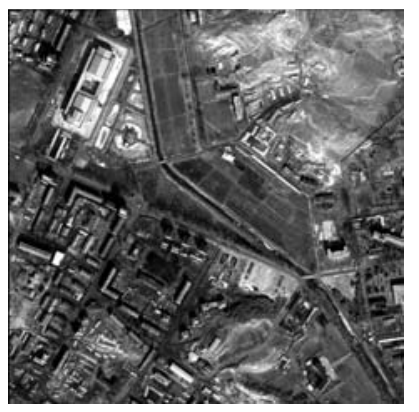

(a)

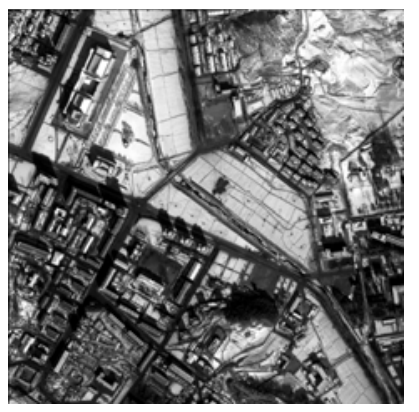

(b)
Figure 1. Panchromatic images of the test area. (a) pan-2006; (b) pan-2010

The HRSS imagery consists of two IKONOS multispectral and panchromatic images acquired in 2006 and 2010(shown in Figure 1). Both data sets are along-track stereo pairs. The multispectral IKONOS imagery has a ground sampling distance (GSD) of $4 \mathrm{~m}$ and contains four bands (red, green, blue, and near infrared). The panchromatic images have a GSD of $1 \mathrm{~m}$. The detail acquisition parameters are shown in Table 1. 
TABLE I. ACQUISITION PARAMETERS OF THE ORIGINAL IKONOS HRSS IMAGERY

\begin{tabular}{|l|l|l|l|l|}
\hline \multirow{2}{*}{} & \multicolumn{2}{|c|}{2006} & \multicolumn{2}{c|}{2010} \\
\cline { 2 - 5 } & \multicolumn{1}{|c|}{ Left Stereo } & \multicolumn{1}{c|}{ Right Stereo } & \multicolumn{1}{c|}{ Left Stereo } & \multicolumn{1}{c|}{ Right Stereo } \\
\hline Data, Time(GMT) & $23 / 02 / 2006,02: 40$ & $23 / 02 / 2006,02: 41$ & $12 / 01 / 2010,02: 29$ & $12 / 01 / 2010,02: 30$ \\
\hline Sensor azimuth(deg) & 7.3730 & 217.6230 & 32.2735 & 122.7043 \\
\hline Sensor elevation(deg) & 65.84380 & 83.2355 & 61.48765 & 79.07821 \\
\hline Sun azimuth(deg) & 157.8630 & 158.1090 & 160.1576 & 160.3700 \\
\hline Sun elevation(deg) & 37.88420 & 37.94370 & 26.19710 & 26.25266 \\
\hline
\end{tabular}

\section{B. DSM Generated with PAN HRSS}

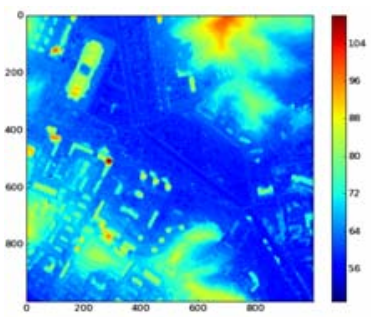

(a)

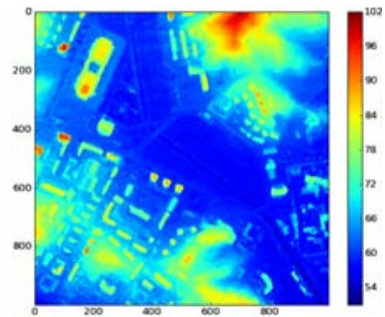

(b)
Figure 2. DSMs of the test data (a) DSM-2006; (b) DSM-2010.

Two DSMs with $1 \mathrm{~m}$ grid resolution are generated using semi-global stereo matching algorithm [9] based on the pan HRSS imagery. The first DSM (referred in the following as DSM-2006) is computed from the IKONOS stereo imagery obtained in 2006, the original generated DSM may contain holes in occluded areas and regions where the matching failed or outliers were removed, which are filled in this paper with SRTM data using the delta surface fill method by Grohman et al [10]. The same method is also applied for the IKONOS imagery acquired in 2010, and the DSM generated is referred in the following as DSM-2010.

\section{Ground Truth Data}

To evaluate the effectiveness of the proposed method for the detection of the position and size of the changed object and the changed situation, in this experiment, we manually extracted the "ground truth" data (shown in Figure 3, where the changed object are highlighted in red), by observing the panchromatic and multispectral imagery of the two years.

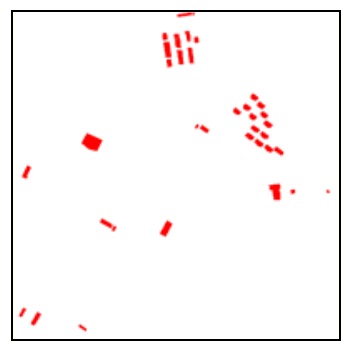

Figure 3. Manually generated ground truth change map

\section{METHODOLOGY}

In this section, the strategy for 3D change detection based on HRSS imagery is presented. The approach consists of four major parts.

\section{A. DSM Co-registration}

To enable the pixels with the same coordinates in the DSM2006 and DSM-2010 to be associated with the same geographical area, a further co-registration between the two resulting DSM is designed and performed to remove any shift in three dimensions between the two DSMs [11].

\section{B. Difference Image Generation and Denoising}

By using the difference based change detection method, DSMs acquired in step-A are subtracted to produce a residual image which represents the height changes between the two dates. To successfully extract the changes from the difference image, denoising is very crucial. In our research, the noise here is mainly caused by shadow (including the cloud shadow) mask and the resulting holes during DSM generation.

1) Shadow. The existence of shadow in HRSS data may cause loss of feature information and false colour tone [12], which will influence the DSMs quality. The shadows in the images are dependent on object position, height and sun elevation. Since the geometry of the two data sets is different, also the shadows position and size are different. To eliminate the negative influence of shadow to the change detection result, the generation of shadow masks is an essential task. In this paper, the shadow detection method proposed by Marchant et.al. [13] shows a high performance in urban area shadow detection.

2) Holes in DSM generation. Even when using dense matching algorithms, due to the variations of the stereo images acquisition conditions and image contrast as well as occluded areas, the generated DSM has some missing information (called holes in the DSM). This is caused by the failed matching. The DSM filling procedure is only for ensuring its continuity. However, the filled value may not express the real situation in some areas. 

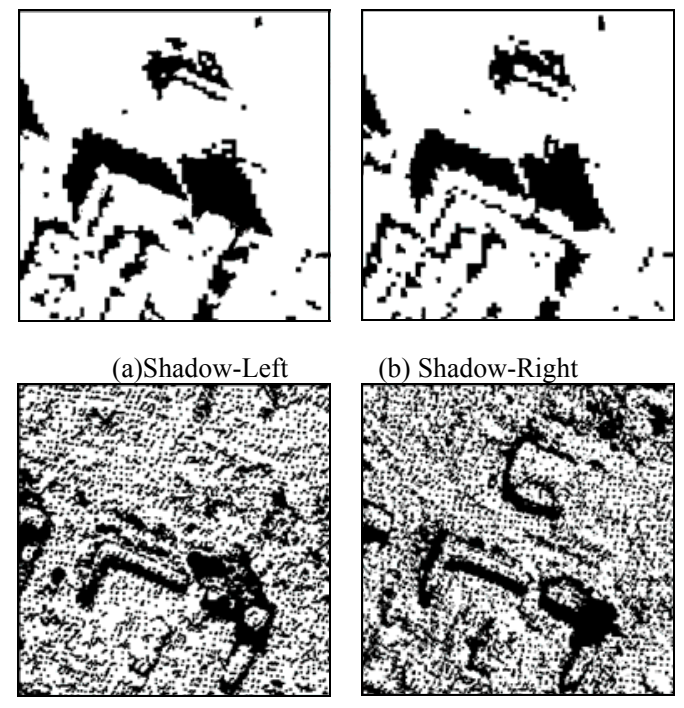

(b) Shadow-Right

(c)Hole-2006

Figure 4. Shadow mask and hole mask

As an example, by comparing the shadow mask (Figure 4 ab) and the holes mask (Figure $4 \mathrm{c}-\mathrm{d}$ ), and also referencing the change detection map and the original multi-spectral imagery, only parts of the shadow area are covered with the holes mask. That demonstrates that the robust characteristic of the DSM generation method we adopt, on the other hand, the holes in the DSM can also be generated by other reasons except the shadow cover. Therefore, we generate the noise mask by intersecting the hole mask and the shadow mask, and then remove the mask area from the difference image, the detail compare-based analyse is showed in section IV.

\section{Thresholding}

Spurious change in altitude (in this case "not-building") can also be caused by other land covers or DSM compute errors. These kinds of changes will influence the building change extraction result, as they are displayed often around the buildings (see Figure 5, the red colour pixels represent the area with changes between 0 to 3 metres). Therefore, thresholding in the difference image is an essential preprocessing task before extracting the "building change" from the "no change background".

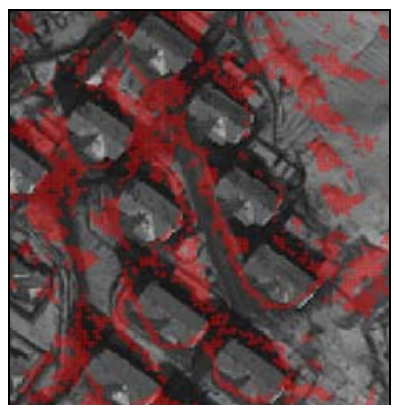

Figure 5. Comparison of the panchromatic image and the small value(0-3 meters) pixels(red).
Many thresholding methods have been developed in the literature, but few are directly applicable to change detection in DSM difference images. For instance, many methods require the distribution of the image intensities to be bimodal. Therefore, in the experimental part of this paper (section IV), we will consider both of manual and automatic thresholding methods, including Otsu[14], Iterative Clusting[15].

\section{Object-based filtering}

After generation of the thresholded image, it is still required to separate "changed building" from the "unchanged background". We therefore consider applying the edgebased building extraction method, and improve the output by filtering the undesired objects based on their properties. In this paper, the object properties that we consider include:

1) Size: It is worth to note that this paper aims at measuring the $3 \mathrm{D}$ changes relative to buildings. Therefore 3 features are used to describe the size of each 3D object.

- Area

- Perimeter

- Height

Here, the object height is defined as the average of the pixels values in the "difference image" belonging to the same changed object, and we exclude all pixels, which have the lowest and the highest $10 \%$ of the height value.

2) Shape: Since size is often not sufficient to identify the true building object from the thresholded image, we consider more shape properties of the objects, including [16]
- Compactness
- Eccentricity
- Convexity

\section{EXPERIMENT AND RESULTS}

\section{A. Experiments overall description}

In this experimental part, we apply the proposed 3D building change detection workflow of section III based on the DSMs generated from HRSS imagery described in section II. First, we subtract the DSM2006 from DSM2010. The difference image is depicted in Figure 6(a) where red indicates large height change values and blue means no change or small height changes values. In this paper, we are interested only in the positive changes. Therefore, only the positive values of the difference image are kept in Figure 6(a). After that, noise is removed by applying the combined shadow-hole mask as described in section III. An Otsu threshold is then applied (shown in Figure 6(b)) to remove DSM computation errors [14]. Objects are then extracted based on the 8-neighbour edge tracking technology (shown in Figure 6(c)). Then we describe the object with size and shape features. The changed areas (see Figure 6(d)) are finally separated based on these properties. 

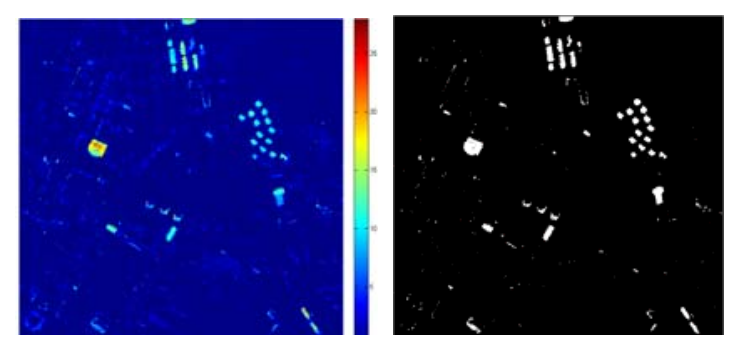

(a) Difference image after denoise (b) Thresholding result

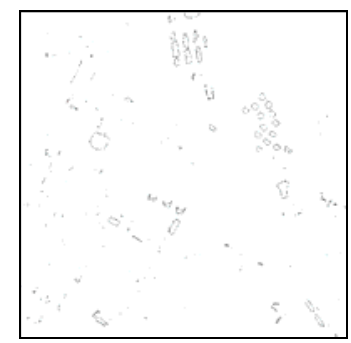

(c) Edge tracking result Figure 6. 3D Change detection procedure

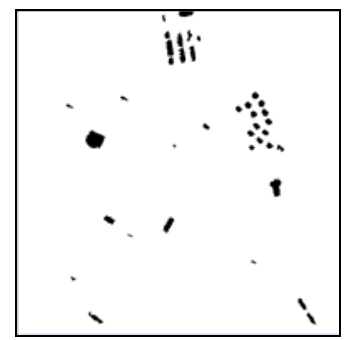

(d) Change detection map

Figure 6 shows one case in the experiments, in which, we use the Otsu thresholding result (with $\mathrm{T}=5.4 \mathrm{~m}$ ), and in the change map extraction step we extracted the objects whose area is larger than $50 \mathrm{~m}^{2}$ and convexity more than 0.7 as the real changes.

\section{B. Quality Measures}

In order to evaluate the effectiveness of the proposed method for the detection of the position and size of the changed objects and the overall change situation, we compare in the following experiment our change detection result with the ground truth data (see Figure 3). The assessment of the change detection results is carried out at both: pixel level and object level.

\section{1) Pixel- based results evaluation}

For the pixel level evaluation, the results are statistics in terms of false alarm, missed alarms, overall errors, and kappa coefficient of accuracy [17]. While the performance of denoising and thresholding are evaluated quantitatively by the ROC curve analysis. [18]

\section{2) Object- based results evaluation}

As a higher level of the analysis, the changed buildings are treated as single objects. In this case smaller or bigger size of the object is not important. In fact, only the effectiveness of the detection of the main regions in the change map is considered in the assessment. Therefore, three parameters are measured to evaluate the object based change detection result:

1) True detected number (TDN): The number of changed objects that are correctly detected as changed.

2) True detected rate (TD): True detected objects number in percentage $\mathrm{TD}=\mathrm{TDN} / \mathrm{N}_{\mathrm{G}} \times 100$.

3) False detected number (FDN): The number of unchanged objects that are incorrectly detected as changed.
4) False detected rate (FD): False detected objects number is percentage $\mathrm{FD}=\mathrm{FDN} / \mathrm{N}_{\mathrm{D}} \times 100$, where $\mathrm{N}_{\mathrm{G}}$ and $\mathrm{N}_{\mathrm{D}}$ are the total changed objects number of the ground truth data and the detected map, respectively.

\section{Experiments}

For the evidence of how each step in our methodology influence the final result, we chose various parameters combinations from both the automatically calculated results and manually given values.

\section{Exp 1, Denoising effect}

To test the effectiveness of the proposed denoising method, we analyze a set of possible solutions, including the original difference image, denoising with only the shadow mask, denoising with only the holes mask, denoising with the union of the hole mask and the shadow mask, and denoising with the intersection of the holes mask and the shadow mask The results in terms of ROC curves with various thresholding value for each denoising method are compared in Figure 7. It shows that denoising with shadow $\cap$ hole mask or just with the shadow mask is nearly equivalent and much preferable to the other methods.

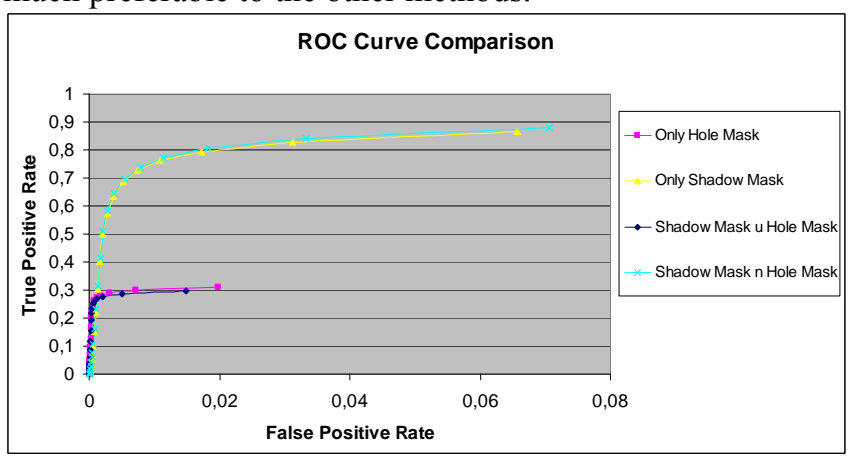

Figure 7. ROC plot comparison between various denoising methods.

\section{Exp 2, Thresholding test}

To enable quantitative assessment of the effect of the thresholding step, we compare the manual thresholding and the automatic thresholding, including Otsu [14] and Iterative method [15] and no thresholding. Since we are trying to reduce the effect of other factors, in this experiment we denoise the difference image with the method designed in Section III and filter the extracted objects with fixed properties (area $>80$ and convexity $>0.75$ ).

Table II and III summarize the pixel and object based evaluation comparison, respectively. From them it can be seen that the selection of $3 \mathrm{~m}$ as the threshold leads to similar results as the Otsu method. Pixel wise the missed alarm is lower for the manual selection but the false alarm rate is lower for the Otsu method. On the other hand this leads to missing one more building in the object based evaluation. More experiments have to be carried out to see if the automatic approach can perform as well as the manual threshold selection. 
TABLE II. PIXEL BASED EVALUATION

\begin{tabular}{|l|c|c|c|c|}
\hline Height & $\begin{array}{c}\text { Missed } \\
\text { alarm } \\
\text { \% }\end{array}$ & $\begin{array}{c}\text { False } \\
\text { Alarm } \\
\text { \% }\end{array}$ & $\begin{array}{c}\text { Overall } \\
\text { Error } \\
\text { \% }\end{array}$ & $\begin{array}{c}\text { Kappa } \\
\text { Accuracy } \\
\text { \% }\end{array}$ \\
\hline $0 \mathrm{~m}$ & 79.86 & 0.99 & 2.28 & 21.23 \\
\hline $2 \mathrm{~m}$ & 44.88 & 0.62 & 1.35 & 56.51 \\
\hline $3 \mathrm{~m}$ & 22.59 & 0.44 & 0.81 & 75.39 \\
\hline Otsu $(5.4 \mathrm{~m})$ & 26.96 & 0.34 & 0.77 & 75.10 \\
\hline Iterative $(1.3 \mathrm{~m})$ & 61.46 & 0.68 & 1.76 & 42.06 \\
\hline
\end{tabular}

TABLE III. OBJECT BASED EVALUATION

\begin{tabular}{|l|l|l|l|}
\hline Height & \multicolumn{1}{|c|}{$\begin{array}{c}\text { True } \\
\text { Detected } \\
\text { \% }\end{array}$} & $\begin{array}{c}\text { False } \\
\text { Detected } \\
\text { \% }\end{array}$ & $\begin{array}{c}\text { True } \\
\text { Detected } \\
\text { Number }\end{array}$ \\
\hline $0 \mathrm{~m}$ & 13.89 & 89.13 & 5 \\
\hline $2 \mathrm{~m}$ & 58.33 & 50.00 & 21 \\
\hline $3 \mathrm{~m}$ & 80.56 & 14.71 & 29 \\
\hline Otsu $(5.4 \mathrm{~m})$ & 75.00 & 15.63 & 27 \\
\hline Iterative $(1.3 \mathrm{~m})$ & 33.33 & 70.00 & 12 \\
\hline
\end{tabular}

Exp 3, Object filter test

In the third experiment, we evaluate the effectiveness of the object properties in the object-based filtering procedure. In that case, we choose the 3 meters height thresholding result, and both of the single properties and combined properties are tested and compared in this experiment.

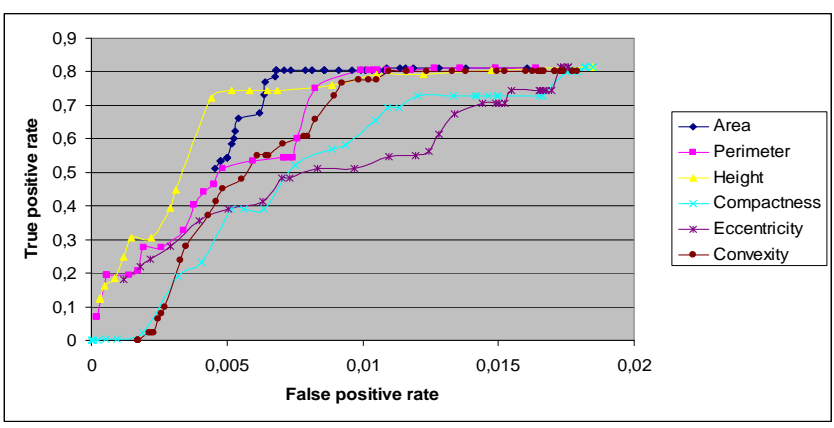

Figure 8. ROC plot comparison between filter parameters

For the single property evaluation, we choose a possible value range (more than 50 possible values for each property) for each parameter, and the accuracy of the filter results are compared through the ROC curve. While the multi parameters based change filter results are recorded with figures (Table VI and V).

Figure 8 shows that the building area performs better among the size features, and convexity is better than eccentricity and compactness. The results of a combined usage of area and convexity parameters are shown in Table IV and V. Table IV shows the variation of area value with a fixed value of convexity (0.75), while Table $\mathrm{V}$ shows the variations for convexity with a fixed value of Area $\left(80 \mathrm{~m}^{2}\right)$. In light gray the optimal parameters are highlighted. It is easy to see that the combine use of area value $\left(80 \mathrm{~m}^{2}\right)$ and convexity value $(0.75)$ as object filter features can get better change detection result.
TABLE IV. Result EVALUATION with FiXed ConveXity Value

\begin{tabular}{|l|l|l|l|l|l|l|}
\hline \multirow{4}{*}{ Area } & \multicolumn{5}{|c|}{ Pixel-based } & \multicolumn{2}{c|}{ Object-based } \\
\cline { 2 - 7 } & $\begin{array}{c}\text { Missed } \\
\text { Alarm } \\
\text { \% }\end{array}$ & $\begin{array}{c}\text { False } \\
\text { Alarm } \\
\text { \% }\end{array}$ & $\begin{array}{c}\text { Overall } \\
\text { Error } \\
\text { \% }\end{array}$ & $\begin{array}{c}\text { Kappa } \\
\text { Accuracy } \\
\text { \% }\end{array}$ & $\begin{array}{c}\text { True } \\
\text { Detected } \\
\text { \% }\end{array}$ & $\begin{array}{c}\text { False } \\
\text { Detected } \\
\text { \% }\end{array}$ \\
\hline 10 & 22.38 & 0.76 & 1.11 & 68.95 & 83.33 & 81.48 \\
\hline 20 & 22.38 & 0.67 & 1.03 & 70.61 & 83.33 & 69.70 \\
\hline 40 & 22.59 & 0.54 & 0.90 & 73.20 & 80.56 & 43.14 \\
\hline 60 & 22.59 & 0.50 & 0.86 & 74.25 & 80.56 & 29.27 \\
\hline 80 & 22.59 & 0.44 & 0.81 & 75.39 & 80.56 & 14.71 \\
\hline 100 & 23.09 & 0.44 & 0.81 & 75.08 & 77.78 & 15.15 \\
\hline 120 & 23.09 & 0.42 & 0.79 & 75.55 & 77.78 & 9.68 \\
\hline 140 & 23.09 & 0.41 & 0.78 & 75.84 & 77.78 & 6.67 \\
\hline 150 & 23.09 & 0.41 & 0.78 & 75.84 & 77.78 & 6.67 \\
\hline
\end{tabular}

TABLE V. Result EVAluation with Fixed AREA VAlue

\begin{tabular}{|c|c|c|c|c|c|c|}
\hline \multirow[b]{2}{*}{ Convexity } & \multicolumn{4}{|c|}{ Pixel-based } & \multicolumn{2}{|c|}{ Object-based } \\
\hline & $\begin{array}{c}\text { Missed } \\
\text { Alarm } \\
\% \\
\end{array}$ & $\begin{array}{c}\text { False } \\
\text { Alarm } \\
\% \\
\end{array}$ & $\begin{array}{c}\text { Overall } \\
\text { Error } \\
\%\end{array}$ & $\begin{array}{c}\text { Kappa } \\
\text { Accuracy } \\
\% \\
\end{array}$ & \begin{tabular}{|c|} 
True \\
Detected \\
$\%$
\end{tabular} & \begin{tabular}{|c|} 
False \\
Detected \\
$\%$ \\
\end{tabular} \\
\hline 0.5 & 20.33 & 1.03 & 1.35 & 65.22 & 83.33 & 45.45 \\
\hline 0.55 & 20.33 & 0.99 & 1.30 & 65.97 & 83.33 & 43.40 \\
\hline 0.6 & 20.33 & 0.93 & 1.25 & 66.89 & 83.33 & 40.00 \\
\hline 0.65 & 20.33 & 0.91 & 1.23 & 67.25 & 83.33 & 37.50 \\
\hline 0.7 & 20.33 & 0.60 & 0.92 & 73.31 & 83.33 & 25.00 \\
\hline 0.75 & 22.59 & 0.44 & 0.81 & 75.39 & 80.56 & 14.71 \\
\hline 0.8 & 39.66 & 0.35 & 0.99 & 66.00 & 61.11 & 24.14 \\
\hline 0.85 & 52.27 & 0.24 & 1.09 & 58.27 & 52.78 & 17.39 \\
\hline 0.9 & 76.17 & 0.10 & 1.34 & 36.30 & 19.44 & 0.00 \\
\hline
\end{tabular}

\section{DISCUSSION}

The three experiments performed to evaluate the proposed 3D change detection approach confirm its effectiveness A detailed discussion of the results given by the three experiments is given in the following:

It is worth noting that in the Exp 1, according to the results shown in Figure 7, the additional use of the hole mask does not bring distinctive advantage when comparing the ROC curves relative to "shadown holes" and "shadow" masks. A better understanding of the improved change detection result is shown in Figure 9. Indeed, in the case of not-flat building roofs, shadows do not exist only around the building, but also on the top of the building, which let the shadow mask destroy some parts of the roof and destroy somehow the overall shape of the building. Therefore the "shadow $n$ hole" mask is preferable even if the ROC curve doesn't show this explicitly.

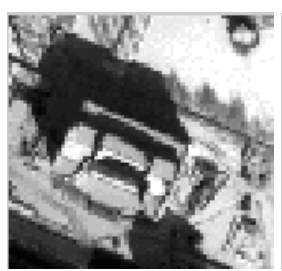

(a)

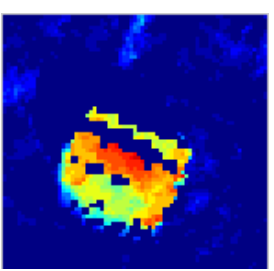

(b)

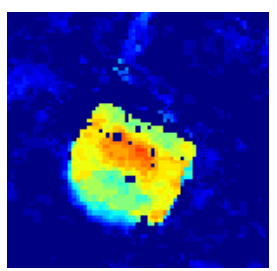

(c)
Figure 9. (a) PAN-2010; (b) Denoising with shadow; (c) denoising with shadow\&holes.

In Exp 2, benefitting from the thresholding, the accuracy of change detection results is clearly increased. On one hand, it 
proves the vital position of thresholding in the proposed change detection procedure. On the other hand, the lower height changes appear to be caused by DSM computation errors but also might be caused by land cover changes, this shows the possibility of monitoring land cover changes with DSMs.

In the third experiment, to evaluate the effectiveness of the proposed object property filter, the ROC curves are compared in Figure 8. It is easy to see, that false positive rates are much lower than in normal cases. This is a direct result of relatively small changes in the whole image. Also in this experiment, it is confirmed that the combined use of height, area and convexity allows a more efficient filter result. In particular, the feature combination that provided the more accurate changed building detection result, is to define single buildings with height higher than 3 meter, area larger than $80 \mathrm{~m}^{2}$ and with convexity more than 0.75 . With these values we come most close to the real situation.

\section{CONCLUSIONS}

In this paper, a novel approach for 3D building change detection is introduced using DSM generated from High spatial Resolution Spaceborne Stereo (HRSS) imagery. Experimental results reported in this paper point out the effectiveness of the proposed approach. It is worth noting that according to the existing automatic DSM generation technology, a proper denoising method and thresholding are essential. The experiments for the object-based filtering demonstrate that the height, area and convexity are the three most important properties. More object properties and property combinations will be tested in future work. As a final remark, it is important to note that, although the approach has been proposed for detecting building changes in urban areas, by shifting the object-based filter properties, it could be used in many other change detection applications.

\section{ACKNOWLEDGMENT}

The authors wish to thank Pablo d'Angelo and Thomas Krauß for contributing their DSM generation method and algorithm.

The present material was produced in the context of the GMOSAIC project, co-funded by the European Commission within the $7^{\text {th }}$ Framework Programme. It is intended only for evaluation purposes and not for redistribution or resale

\section{REFERENCES}

[1] D. Lu, P. Mausel, E. Brondizio and E. Moran, "Change Detection Techniques," International Journal of Remote Sensing, 25 (12), pp 2365-2407, 2004.

[2] L. Zhang, "Automatic Digital Surface Model (DSM) generation from Linear array images," Ph.D. Thesis, Institute of Geodesy and Photogrammetry, ETH Zurich, Switzerland, 2005.
[3] D. Akca, "Least Squares 3D surface matching," Ph.D. thesis, Institute of Geodesy and Photogrammetry, ETH Zurich, Switzerland, 2007.

[4] T. Krauß, P. Reinartz, and U. Stilla, "Extracting Orthogonal Building Objects in Urban Areas from High Resolution Stereo Satellite Image," International Archives of Photogrammetry, Remote Sensing and Spatial Information Sciences, Pairs, 2007, 36(3/W49B).

[5] P. d'Angelo, M. Lehner, and T. Krauss, "Towards automated dem generation from high resolution stereo satellite images," International Society for Photogrammetry and Remote Sensing, pp. 1137-1342.

[6] A. Singh, "Digital change detection techniques using remotelysensed data," International Journal of Remote Sensing, 10 (6), pp 989-1003, 1989.

[7] T. Fung, "an assessment of TM imagery for land-cover change detection," IEEE Transactions on Geoscience and Remote Sensing, vol. 28 , No. 12,1990

[8] L. Bruzzone, and D.F. Prieto, "Automatic Analysis of the Difference Image for Unsupervised Change Detection," IEEE Transactions on Geoscience and Remote Sensing, vol. 38, No. 3, May 2000.

[9] H. Hirschmüller, "Stereo processing by semiglobal matching and mutual information," IEEE Transactions on Pattern Analyses and Machine Intelligence, 30 (2), Feb. 2008.

[10] G. Grohman, G. Kroenung, and J. Strebeck, "Filling SRTM voids: The delta surface fill method," Photogrammetric Engineering and Remote Sensing 72(3), pp. 213-216, 2006.

[11] X. Dai, and S. Khorram, "the effects of image misregistration on the accuracy of remotely sensed change detection," IEEE Transactions on Geoscience and Remote Sensing., vol. 36, No. 5, pp. 1566-1577 Sep. 1998.

[12] V. J. D. Tsai, "A comparative study on shadow compensation of color aerial images in invariant color models," IEEE Transactions on Geoscience and Remote Sensing, vol. 44, No. 6, June 2006.

[13] J.A. Marchant and C.M. Onyango, "Shadow-invariant classification for scenes illuminated by daylight," J. Opt. Soc. Am. A 17,pp.1952-1961, 2000.

[14] N. Otsu, "A threshold selection method from gray-level Histograms," IEEE Transactions on Systems Man Cybernet, 9, pp. 62-66, 1979

[15] T. Ridler, and S. Calvard, "Picture thresholding using an iterative selection method", IEEE Transactions on Systems Man Cybernet. 8, pp. 629-632, 1978.

[16] B. M. Mehtre, M. S. Kankanhalli, W. Lee, "Shape measures for content based image retrieval: A comparison Information Processing \& Management," Volume 33, Issue 3, pp.319-337, May 1997. 
[17] R. Congalton, "A review of assessing the accuracy of classification of remotely sensed data," Remote Sensing of Enviroment., Vol. 37, Issue 1, pp. 35-46, July 1991,

[18] T. Fawcett, "An introduction to ROC analysis", Pattern Recognition Letters, vol. 27, pp 861-874, 2006. 